\title{
All in the mind? The neural correlates of unexplained physical symptoms
}

\author{
Sean A. Spence
}

\begin{abstract}
Physical symptoms with no medical explanation are commonly experienced by healthy people and those attending clinics. Psychiatrists see such patients in liaison settings and clinics for those with psychotic and affective disorders. The pathophysiology remains obscure; physical investigations are usually performed to exclude pathology rather than elucidate dysfunction. However, modern neuroimaging has allowed the study of nervous system structure and function. Although there are few diagnostically specific findings, patterns of association have emerged: where action is impeded (certain forms of conversion disorder and chronic fatigue syndrome) frontal systems of the brain are often implicated; when subjective awareness of the body is disturbed (passivity phenomena and anorexia nervosa) temporo-parietal cortices appear to be dysfunctional. The caudate nuclei (components of the frontal executive circuit) are implicated in a variety of syndromes (including body dysmorphic disorder, somatisation and chronic fatigue). The brain may be viewed as a cognitive neurobiological entity, crucially oriented towards action (for survival). Psychiatric syndromes that have an impact on bodily awareness signal dysfunction within systems representing that body and its performance in time and space.
\end{abstract}

Medically unexplained physical symptoms are common among healthy people and those attending clinics. About $20-30 \%$ of people seen in general practice lack a demonstrable cause for their symptoms (Rosendal et al, 2005) and the figures are higher still in general hospitals (Talley, 1998; Stone \& Zeman, 2001). Although liaison psychiatrists encounter some of these patients, other clinicians manage most of them, confronting the attendant risks of over-investigation, misdiagnosis and inappropriate treatment. Many psychiatric syndromes can be associated with unexplained physical phenomena (Box 1), and these vary greatly in their phenomenology, proposed aetiology and appropriate management. What these syndromes share is an irreducible, subjective core: only the patient can fully describe their symptom, there is no external reference; that is, with symptoms such as pain or somatic hallucination, there is nothing for the physician 'to see' (Berrios, 1982).

It may seem paradoxical to set about investigating the physical basis of symptoms that are regarded as 'medically unexplained' since their classification hinges upon their having no explanation and, hence, by implication, no biological basis. However, such a view fails to acknowledge the constant interaction between 'states of mind' and 'states of the body' in a cognitive neurobiological system - the brain. It also risks minimising the patient's reality, a lived experience that is 'embodied' (Butler et al, 2004). Whether the symptom is numbness or fatigue, awareness of that symptom and its emotional sequelae are proposed to be represented within the central nervous system, and even a deliberately feigned disorder depends on neurological processes for its 'performance' (Spence et al, 2000). Hence, although it might be reasonable to state that some disorders lack a demonstrable lesion (e.g. a tumour), it seems unlikely that they will lack any relationship to the state of the patient's brain.

\section{Cognitive neurobiological architecture}

Before embarking on a review of the sites of dysfunction implicated in specific disorders it is appropriate to hypothesise where such symptoms might arise. Which brain systems represent the ongoing state of the body? This is a complex question and is the subject of much contemporary research.

Sean Spence is Professor of General Adult Psychiatry at the University of Sheffield (Academic Clinical Psychiatry, University of Sheffield, The Longley Centre, Norwood Grange Drive, Sheffield S5 7JT, UK. Email: s.a.spence@sheffield.ac.uk). He studied medicine at Guy's Hospital, London, then worked in several medical specialties before training in psychiatry at London's Charing Cross Hospital, undertaking a Medical Research Council training fellowship at Hammersmith Hospital and a De Witt Wallace travelling fellowship to Cornell University, New York. His research concerns the cognitive neurobiology of volition. 
Box 1 Some psychiatric syndromes that have an impact on bodily experience (ICD-10 diagnostic codes are in parentheses)

- Paranoid schizophrenia (F20.0) - passivity phenomena, somatic hallucinations

- Schizoptypal personality (F21) - bodily illusions, dysmorphophobic ruminations

- Persistent delusional disorder (F22) hypochondriacal (somatic) delusions, dysmorphophobia, delusional parasitosis

- Severedepression with psychotic symptoms (F32.3) - hypochondriacal delusions, Cotard's delusion

- Dissociative (conversion) disorder (F44) - includes motor signs (44.4), convulsions (44.5) and sensory loss (44.6)

- Somatoform disorder (F45) - includes somatisation disorder (45.0) and hypochondriacal disorder (45.2) - would also subsume Briquet's syndrome

- Persistent somatoform pain disorder (F45.4)

- Neuraesthenia (F48) - fatigue syndromes

- Anorexia nervosa (F50.0) - disturbed body image

In simple terms, there is a distinction between the frontal lobes of the brain, anterior to the central sulcus, and the posterior cortices: the parietal, temporal and occipital lobes. The latter receive incoming sensory information from the major sense organs, via the thalamus, whereas the frontal systems are responsible for predicting, planning, programming and executing actions in the world (and also in 'the mind'; Spence \& Frith, 1999). All these systems are interconnected, so that even a simple act is the product of motor initiation, sensory expectation and feedback. Subcortical structures also play an important role: the frontal lobes contain at least five semi-closed loops, which funnel information from specific cortical fields to targeted regions of the striatum (caudate and putamen), on into the thalami, whence they are closed by further projections, returning to the cortex (Alexander \& Crutcher, 1990). The 'motor' loop focuses primarily on the supplementary motor area, an area of premotor (programming) cortex high up on the medial surface of the frontal lobes; the 'executive' loop projects to the dorsolateral prefrontal cortex, an area implicated in working memory, planning and novelty generation. The latter circuit also incorporates the caudate nucleus, a component of the striatum that is involved in planning, reward and emotional processing (Fig. 1).
Although the sense organs send data to the primary sensory areas in the parietal (somatosensory), temporal (auditory) and occipital (visual) cortices for preliminary analyses, more complex computations occur elsewhere. Hence, there are streams of data leaving the occipital cortices along the ventral ('what is it?') and dorsal ('where is it?') pathways, to the temporal and parietal cortices respectively. Within the temporal cortices there are regions concerned with semantic knowledge - the identity and meaning of stimuli. There are heteromodal regions of the parietal cortex that are engaged in the analysis and synthesis of somatic, auditory and visuospatial information with reference to egocentric, bodily oriented coordinates.

We might predict that certain disorders of bodily experience would involve such systems, particularly those within the parietal and temporal cortices (i.e. those that may be grossly simplified as dealing with bodily 'space' and 'meaning' respectively). We might also be more specific and differentiate somatic (pertaining to bodily surface and voluntary musculature) from visceral (bodily interior and involuntary musculature) sensations. It has been suggested that one reason visceral (as opposed to somatic) sensations are so difficult to localise within the body is that their projections are not focused on the primary somatosensory strip (with its familiar homunculus, representing localised somatic sensation) but instead more diffusely distributed, primarily to the secondary somatosensory cortices and limbic regions (Schnitzler et al, 1999; Aziz et al, 2000). This is illustrated by the pain associated with appendicitis: vaguely central abdominal (visceral) pain precedes specific localisation of pain to the right iliac fossa, following peritoneal (somatic) involvement.

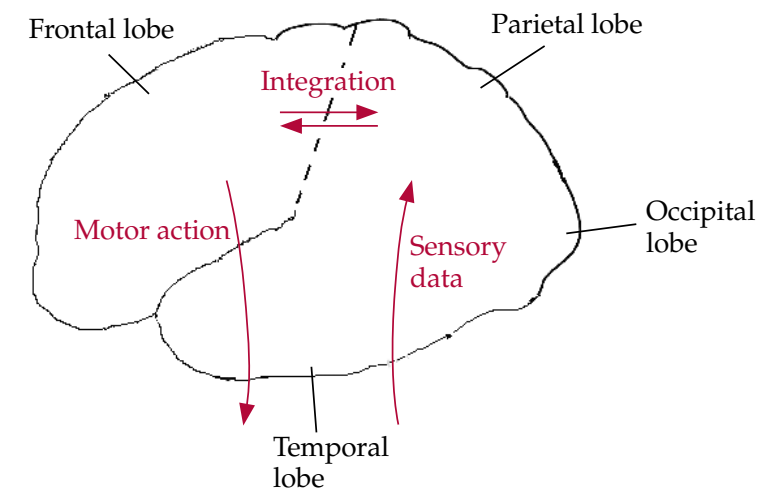

Fig. 1 Cartoon illustrating a much-simplified cognitive architecture of the frontal and posterior brain systems' utilisation of incoming sensory data. The initiation of voluntary actions implicates frontal systems (see text for details). 
Furthermore, the simple association of frontal regions with motor systems and posterior regions with sensory systems breaks down when we consider emotional states of the body. Medial frontal regions (including the anterior cingulate cortex) are particularly concerned with subjective awareness of pain and emotional experience (Peyron et al, 2000; Phan et al, 2002), whereas the basic emotions, such as fear and disgust, involve other limbic foci (in particular amygdala and caudate respectively) (Calder et al, 2001; Phan et al, 2002). Such systems modulate autonomic arousal and responsivity and are implicated in conditions such as panic disorder and obsessive-compulsive disorder (OCD). Emotional processing also has an impact on relationships with others because it is linked to the ability to recognise their distress. This suggests that amygdala and medial prefrontal dysfunction might underlie certain aspects of psychopathy (Damasio, 2000; Blair, 2003).

\section{Imaging techniques}

Magnetic resonance imaging (MRI) provides a safe means of assessing the structure of the living brain. Other techniques can detect local or diffuse changes in brain activity. They include those that utilise radiotracers (positron emission tomography - PET - and single-photon emission computed tomography -SPECT) and those that use MRI (such as functional magnetic resonance imaging - fMRI). These techniques are often used to detect proxy markers of synaptic activity; for example, changes in regional cerebral blood flow in certain forms of PET and SPECT, and changes in blood oxygen level-dependent response in $\mathrm{fMRI}$. Techniques that use radiotracers (PET and SPECT) may also be used to measure regional brain metabolism (e.g. with radiolabelled glucose molecules) and neurotransmitter receptor binding (with radiolabelled ligands). Hence, there is a wide range of methods that may be applied in the investigation of the biology of neuropsychiatric disease, although these vary in their availability and the ease with which they can be used in nonspecialised centres.

\section{Syndromes affecting bodily experience}

\section{Passivity phenomena}

Central to the concept of schizophrenia is a breach of physical borders, which we often interpret in mental terms: the dissolution of ego boundaries exemplified by the Schneiderian first-rank symptoms (Schneider, 1974). The patient with passivity phenomena, 'made' thoughts or movements, believes that their autonomy is being usurped by outside forces (Mullins \& Spence, 2003). A spirit or persecutor controls their movements. Their thoughts are directly accessed by others.

From which brain systems might this sensation arise? Study of patients with neurological damage has suggested that damage to the parietal lobes might produce similar phenomena (Spence, 2001; Spence \& Halligan, 2002). One important theoretical account of first-rank symptoms attributes them to a failure in the monitoring of ongoing action; more specifically, to a failure by the brain's motor programming systems to predict the future sensory consequences of action (Frith et al, 2000). By 'forward modelling', the brain predicts its own outcome states following future acts. If I lift my arm, my brain has already computed the sensory stimuli arising from such an act. A deviation from such predictions alerts the brain to potential errors and the need for correction. However, if there is a fault within the system then my action might feel as if it had come from 'nowhere', as if an outside force were responsible.

Although sophisticated cognitive psychological experiments have investigated whether people experiencing first-rank symptoms are aware of their actions (Frith \& Done, 1989), one of the advantages of functional neuroimaging techniques is that they allow investigators to examine the motor system directly and to locate functional abnormality. In one such study, Spence and colleagues (1997) used PET to examine the brain activity associated with moving a joystick in freely chosen directions by people with schizophrenia experiencing passivity ('made' thoughts and actions), other people experiencing psychosis but not passivity phenomena, and healthy controls. Patients with passivity exhibited relative hyperactivation of the right inferior parietal cortex, an area implicated in spatial awareness and programming, and the left supplementary motor area, a region of premotor cortex that is also involved in the programming of action. Hence, the findings are consistent with the view that systems engaged in programming action in space are disturbed in people experiencing passivity (their disturbance remitting with symptomatic recovery) (Spence et al, 1997).

Subsequent studies have also found parietal abnormalities in patients with such symptoms (Franck et al, 2002). In one recent study hyperactivation of the right parietal cortex was found while such patients performed self-paced finger movements in an MRI scanner (Ganesan et al, 2005; Fig. 2). Another study has shown that people with schizophrenia reporting motor passivity phenomena ('made' movements) exhibit reductions in right parietal and left prefrontal grey matter volume relative to other people with schizophrenia (Maruff et al, 2005). Moreover, a study 


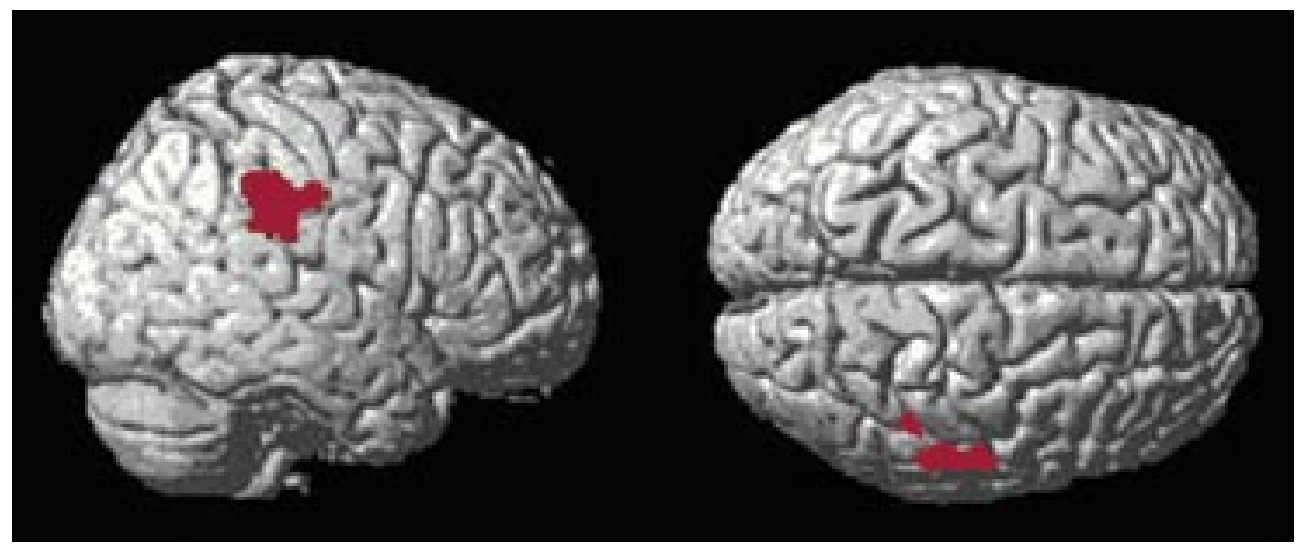

Fig. 2 Aberrant hyperactivation of right parietal (sensory) regions (Brodmann area 40) during action initiation by people experiencing first-rank symptoms of schizophrenia (Talairach coordinates =57, $-29,36 ; T=4.7 ; P<0.0001$ (uncorrected); family-wise error corrected $P=0.015)$. Data derived from Ganesan et al (2005).

of students with high schizotypy scores revealed that those who feared losing control of their thoughts had reduced grey matter volume of the supplementary motor area (Matsui et al, 2002).

Hence, current studies on passivity phenomena suggest that the parietal cortices (which are concerned with spatial and bodily awareness) and key planning and programming areas within the frontal lobes are implicated in the disturbed sense of agency and bodily awareness.

\section{Somatic hallucinations and delusions}

AnfMRIstudy of a36-year-old man with schizophrenia who was experiencing somatic hallucinations (that he was 'touched by spirits') revealed an association with activation of corresponding primary somatosensory and posterior parietal cortices (Shergill et al, 2001). These findings were consistent with those of an earlier electroencephalographic study of a similar patient (Baldeweg et al, 1998). Again, the suggestion is that sensory and association cortices are implicated in the generation of complex abnormalities of bodily experience encountered in schizophrenia. Note that the patient's phenomenology implicated the surface of the body ('touched by spirits'), which is in line with the primary somatosensory cortex being activated.

There are several interesting case reports from Japan on neuroimaging in patients with delusional parasitosis. Maeda and colleagues (1998) described an elderly man who felt worms moving around in his mouth. His MRI scan revealed an old infarction of the right putamen (an area receiving parietal afferents); his symptoms resolved with pimozide. Wada et al (1999) performed a SPECT investigation before and after response to clomipramine of an elderly woman who believed 'something was moving in her abdomen'. At the height of her symptoms she exhibited hypoperfusion of left temporo-parietal cortices.

Similarly, Ota and colleagues (2003) used SPECT to investigate the brain function of an elderly man before and after modified electroconvulsive therapy for somatic (nihilistic) delusions: 'Food disappears inside of my oesophagus', 'my jaw comes off'. This man exhibited hypoperfusion of left parietal and temporal regions, which improved following treatment. The same regions were implicated in an elderly woman who complained of oral infestation, which responded to paroxetine (Hayashi et al, 2004).

Patients with Cotard's delusion of negation are usually depressed and come to believe that they are dead or decaying. There have been rather disparate accounts of functional anatomical correlates of Cotard's delusion using SPECT. Hashioka et al (2002) described a patient with bilateral hypofrontality, which resolved on recovery; De Risio and colleagues (2004) found no focal perfusion deficit in a patient with more classical symptomatology ('he had no internal organs and was already dead') but did find a reduction in striatal $D_{2}$ receptor binding, which is consistent with excessive endogenous dopamine at the time of psychosis. Gardner-Thorpe \& Pearn (2004) described two further patients: one with associated arteriovenous malformations in the parietal lobes and the other with multiple sclerosis. Despite multiple sclerosis of 20 years' duration the latter patient described recent onset of transient psychotic episodes. During such episodes she felt as if 'her flesh were falling off', 'as if there were liquid inside her that was draining out'. 'Whilst experiencing the symptom she believed it was really happening but next morning had gained a somewhat perplexed 
insight into its hallucinatory nature' (Gardner-Thorpe \& Pearn, 2004: p. 564). This case report exemplifies the blurring of phenomenological boundaries seen in certain accounts of somatic hallucinations, Cotard's delusion and, indeed, hypochondriacal delusions.

\section{Dysmorphophobia}

There is little recent work devoted to the onset of disturbed bodily awareness (dysmorphophobia), where the central feature is preoccupation with slight or non-existent defects in appearance, causing significant distress or impairment in functioning. About half of patients with body dysmorphic disorder experience psychosis, although there is also a phenomenological similarity to OCD, exemplified by repeated checking and ritualised behaviours. Gabbay and colleagues (2003) described a 24-yearold man who developed a preoccupation with the width of his nose following encephalitis; structural MRI revealed marked left-sided fronto-temporal atrophy.

The emergence of compulsive or ritualistic behaviours has been linked to both temporal lobe and basal ganglia pathology. There is a theory that specific basal ganglia circuits might be involved in OCD-spectrum disorders, with those disorders with cognitive phenomenology (OCD and body dysmorphic disorder) involving the caudate and those characterised by motor abnormalities (Tourette syndrome and trichotillomania) the putamen (Rauch et al, 2003). In this regard it is of interest that an MRI study of eight middle-aged women with body dysmorphic disorder revealed leftward shift of caudate asymmetry and increased white matter volume. However, apart from being a marker of striatal abnormality it is unclear how such an asymmetry might disturb bodily awareness. A SPECT study of brain function in six people with body dysmorphic disorder found a broad range of abnormalities that implicate the parietal lobes (Carey et al, 2004). This is consistent with the role of the latter in supporting bodily awareness.

\section{Eating disorders}

Concern with the body's size and shape is a hallmark of the eating disorders, aspects of which seem to imply a misperception of bodily form. Is there evidence in favour of abnormal cerebral processing of the embodied self? Neuroimaging studies in people with eating disorders are surprisingly few, but already there are some interesting leads.

A study of three people with anorexia nervosa who were confronted with images of their own bodies revealed right amygdala activation, which is consistent with the latter's role in processing negative affect (Seeger et al, 2002). Although this finding awaits replication, a later study of a larger sample revealed that people with anorexia and/or bulimia exhibited reduced activation of right temporo-parietal regions (those concerned with bodily awareness and recognition), in comparison with healthy controls, when confronted with images of standard female forms (Uher et al, 2005). The authors concluded that functional abnormalities in specific regions might underlie abnormal self-perception in these disorders. The importance of right-hemisphere abnormalities in eating disorders is also supported by a recent review. Uher \& Treasure (2005) report that although simple changes in appetite and eating behaviour occur with hypothalamic and brain-stem lesions, more complex disturbances, resembling the psychopathology of idiopathic eating disorders, arise more commonly following right frontal and temporal damage.

Recent PET studies of people recovering from anorexia have revealed abnormalities of central serotonergic and dopaminergic function (Frank et al, 2002, 2005).

\section{Conversion syndromes ${ }^{t}$}

Conversion syndromes are characterised by abnormalities of voluntary movement and subjective report (e.g. hemi-anaesthesia) that cannot be explained by demonstrable cerebral pathology. The onset of the disorder is said to coincide with emotional distress and the symptoms themselves are inconsistent (e.g. paralyses may wax and wane, normal function may be elicited by distraction or sedation; Spence, 1999). To date, there have been a few neuroimaging studies of single patients or small series of patients with conversion syndromes using SPECT, PET or fMRI. Results may have been influenced by confounding variables such as the presence of comorbid diagnoses (often depression and/or personality disorder) and symptom chronicity (not least since many such studies are from tertiary centres, which attract patients with atypical, chronic disorders). I have reviewed the pros and cons of these approaches elsewhere (Spence, 1999, 2006).

Nevertheless, a common theme has emerged (Box 2): sensory deficits (e.g. anaesthesia or blindness) are associated with hypoactivation of the relevant sensory cortices and evidence of disturbed function in 'higher' frontal regions (Tiihonen et al, 1995; Yazici

\footnotetext{
${ }^{\dagger}$ For a more detailed consideration of conversion syndromes in APT, see Owens, C. \& Dein, S. (2006) Conversion disorder: the modern hysteria. Advances in Psychiatric Treatment, 12, 152-157. Ed.
} 
Box 2 Common themes from neuroimaging studies of conversion syndromes

- Sensory deficits (anaesthesia or blindness) are associated with hypoactivation of the sensory cortices and disturbed function of 'higher' frontal regions (Tiihonen et al, 1995; Yazici \& Kostakoglu, 1998; Vuilleumier et al, 2001; Mailis-Gagnon et al, 2003; Werring et al, 2004)

- Motor disturbance is associated with either excessive activation of (inhibitory) orbitofrontal cortices (Marshall et al,1997) or suppression of activation in the dorsolateral prefrontal cortex (Spence et al, 2000)

\& Kostakoglu, 1998; Vuilleumier et al, 2001; MailisGagnon et al, 2003; Werring et al, 2004); whereas motor disturbance is associated with either excessive activation of (inhibitory) orbitofrontal cortices (Marshall et al, 1997) or suppression of activation in the dorsolateral prefrontal cortex (an area involved in action generation) (Spence $e t$ al, 2000). A common view is that lower centres are somehow inhibited or suppressed by higher centres. There has been debate as to whether these findings may adequately distinguish conversion syndromes from feigning (Marshall et al, 1997; Spence, 1999). Nevertheless, there is the hope that functional neuroimaging techniques may (in the future) help to establish an empirical framework for conversion syndromes.

\section{Somatisation}

Somatisation refers to recurrent, multiple somatic complaints which cannot be attributed to demonstrable organic pathology. It has been postulated to be a consequence of disturbed attention, so that people with somatisation are unable to filter out irrelevant somatic sensations. There are few neuroimaging studies of people with somatisation.

Garcia-Campayo et al (2001) used SPECT to examine a series of people with somatisation who were without comorbid Axis I diagnoses. Visual inspection of the scans (there was no control group) revealed that most of their 11 participants had evidence of focal hypoperfusion, predominantly of the frontal, cerebellar and temporo-parietal regions.

$\ddagger$ A cognitive-behavioural intervention for somatoform disorders has been described by Patel, V. \& Sumathipala, A. (2006) Psychological approaches to somatisation in developing countries. Advances in Psychiatric Treatment, 12, 54-62. Ed.
Subsequently, Hakala and colleagues (2002) used PET to compare brain function of women with chronic somatisation with that of a control group. The women with somatisation exhibited reduced metabolism of specific subcortical regions, including bilateral caudate nuclei. The volume of these caudate nuclei (in the same women) was found to be enlarged by MRI (Hakala et al, 2002, 2004).

\section{Chronic fatigue syndrome}

Fatigue can be defined as difficulty in initiating or sustaining voluntary activities, and may be caused by central or peripheral neurological or systemic factors (Chaudhuri \& Behan, 2004). Chronic fatigue syndrome is characterised by a sense of fatigue that persists for at least 6 months and is unattributable to other physical or psychiatric disorder. It provides another example of a diagnosis of exclusion (similar to conversion and somatisation), resting very much on the patient's subjective account. Nevertheless, it is clinically desirable to perform structural MRI of the brain in patients with chronic fatigue, not least because persistent fatigue may be a presenting symptom of multiple sclerosis (Lange et al, 1998).

There have been many neuroimaging studies of people with chronic fatigue over the past decade and in recent years a range of scanning methods and techniques for data analysis have been used. Early studies suggested that structural, white matter defects might be more common in patients with chronic fatigue syndrome, particularly within the frontal lobes (Natelson et al, 1993; Lange et al, 1999; Cook et al, 2001), but this was not demonstrated by others (Cope et al, 1995; Greco et al, 1997). The defects most often cited were so-called hyperintensities, possibly reflecting vascular pathology; these are often reported to increase with age in healthy people (and also to be over-represented in a number of psychiatric disorders).

Although focal functional deficits were also reported in early studies, a very well-controlled twin study of patients with chronic fatigue syndrome and their discordant (i.e. healthy) co-twins revealed no abnormalities of resting-state regional cerebral blood flow which were specific to the disorder (Lewis et al, 2001). Hence, there may be no trait resting-state functional abnormalities that adequately characterise all patients with chronic fatigue syndrome. Some investigators have refined their samples to focus on specific features of the disorder, e.g. late-onset syndrome or cognitive impairment. Others have focused on 'cognitive challenges': studying functional response while patients perform a cognitive task.

Siessmeier and colleagues (2003) conducted the first PET study of cerebral metabolism in chronic 
fatigue in which the data were analysed using an observer-independent technique (i.e., not a manually determined region-of-interest analysis, which may be subject to observer bias). They found that, despite sample heterogeneity, participants demonstrated reduced activity in the orbitofrontal and anterior cingulate cortex, regions implicated in the control (inhibition and monitoring) of behaviour. However, there was no correlation with fatigue per se.

De Lange et al (2004) used fMRI to study people with chronic fatigue who were attempting motor and visual imagery tasks. Under both conditions participants exhibited hypofunction of the caudate nuclei, whereas on error trials they failed to activate ventral regions of the anterior cingulate cortex (implicated in affect regulation).

In a structural MRI study, Okada and colleagues (2004) used an automated, data-led approach to reveal bilateral reductions of cortical grey matter in dorsolateral prefrontal regions. The deficit in the right dorsolateral prefrontal cortex correlated with the severity of symptomatic fatigue. This area had also been implicated in an earlier SPECT study of late-onset chronic fatigue (Goldstein et al, 1995).

Turning to neurochemistry, Cleare et al (2005) summarised the evidence that fatigue may be associated with excessive serotonergic tone and demonstrated, using a ligand for the 5HT-1A receptor, that untreated patients without comorbid diagnoses had reduced 5HT-1A binding occupancy on PET scans. This finding would be compatible with reduced numbers of receptors or their decreased affinity for the ligand (which would be in accordance with a central disturbance of serotonergic function in chronic fatigue syndrome). There were no correlations with fatigue per se, although the patients studied were at, or close to, ceiling on their fatigue severity ratings. Hence, there may have been insufficient withingroup variance to detect such a correlation.

\section{Pain syndromes}

Pain is processed within a distributed 'pain matrix' involving lateral systems, incorporating the lateral thalamic nuclei, insulae and secondary somatosensory cortices (concerned with pain localisation), and medial systems, incorporating the midline and intralaminar thalamic nuclei, amygdalae and anterior cingulate cortex (which are more concerned with its affective significance) (Ladabaum et al, 2000; Peyron et al, 2000; Jones et al, 2003; Vogt, 2005). There is also a distinction between visceral and somatic pain: the former eliciting most response from within the medial system and the latter from the lateral system (Dunckley et al, 2005). In general, visceral pain evokes greater 'unpleasantness' than somatic pain, even when 'pain' levels are controlled for. These phenomena differ in other respects: visceral pain elicits immobilisation whereas somatic pain is more likely to precipitate a fight/flight response (hence the greater involvement of motor regions) (Ladabaum et al, 2000; Dunckley et al, 2005, Vogt 2005). Visceral pain is likely to be harder to localise (Schnitzler et al, 1999).

\section{Functional pain}

The literature on functional pain syndromes such as irritable bowel syndrome, fibromyalgia and atypical facial pain has been reviewed by Derbyshire (2003). A coherent message has yet to emerge, although there are interesting patterns. For instance, chronic functional pain might be associated with increased responsiveness of the anterior cingulate cortex, whereas chronic organic (specifically, inflammatory) pain might be associated with downregulation of the anterior cingulate cortex and thalamus (Peyron et al, 2000). In organic disease, adequate pain relief elicits increased activation of the latter foci (Peyron et al, 2000; Vogt, 2005). The highest concentration of endogenous opioid receptors is found in the midanterior cingulate cortex, which is in accordance with this region's functional response to opiate analgesia and placebos (Peyron et al, 2000; Vogt, 2005).

\section{Angina pain}

Angina pain is associated with activation of the anterior cingulate cortex and thalamus (and prefrontal foci). However, 'silent' ischaemia activates the thalamus but does not activate the anterior cingulate cortex (Ladabaum et al, 2000). This suggests that subjective awareness of pain is crucially linked to activity within the anterior cingulate cortex (consistent with its specific response to successful analgesia) (Jones et al, 2003; Vogt, 2005). It also suggests that the thalamus may play a role in gating the conscious perception of pain (Ladabaum et al, 2000). Overactivity of the anterior cingulate cortex in functional pain syndromes may therefore reflect the role of higher centres, perhaps manifesting increased vigilance towards visceral sensation (Aziz et al, 2000; Ladabaum et al, 2000; Derbyshire et al, 2004). A detailed account of the anatomy of the anterior cingulate cortex and regional specialisation is provided by Vogt (2005).

\section{Conclusions}

The advent of neuroimaging techniques has led to a great many studies aimed at discovering the biological correlates of a variety of somatic phenomena, from 
the frankly psychotic (e.g. delusional parasitosis) to the traditionally 'neurotic' (e.g. somatisation). At present, such studies are largely correlational and, although they implicate certain brain foci, they have yet to provide detailed accounts of pathophysiology.

Perhaps the most chastening lesson from this review is the striking lack of specificity of any of the findings so far described. Although we might predict that a patient describing bodily disturbance of 'some kind' will exhibit abnormalities of certain candidate brain regions, we would have great difficulty modifying their diagnosis or treatment on the basis of the results of brain scanning.

Also, the phenomenological boundaries between conditions are conspicuously porous (Cahill \& Frith, 1996): for example, where does thought insertion end and somatic hallucination begin if a person can point to the site of entry of an alien thought? The comorbidity inherent in many of the physical syndromes makes it correspondingly difficult to obtain 'pure' samples (e.g. of people with conversion syndrome or somatisation); and, were they to be obtained, one might ask how representative they were of the population experiencing the syndrome. One might conclude that the diagnostic categories available to us do not constitute 'natural kinds'. There is too much phenomenological overlap.

Hence, it is perhaps unsurprising that many of the same brain regions are implicated across different psychiatric syndromes. This survey reveals that the frontal lobes are often implicated where there is a failure of action, as in conversion disorder or chronic fatigue syndrome, whereas temporal and parietal lobes are often implicated in those disorders affecting the individual's awareness of their own subjective space (e.g. passivity phenomena or anorexia nervosa). Several disorders (body dysmorphic disorder, somatisation and chronic fatigue syndrome) implicate dysfunction of the caudate nuclei, areas of the striatum contributing to cognitive, motor and emotional control. A full description of such syndromes will require understanding of the principles of regional cognitive neurobiology that is not confined to cortical foci but incorporates distributed networks and systems.

From a technical point of view it is possible that the literature surveyed may be biased in several ways. Among case reports, a positive finding with a plausible focal activation is perhaps more likely to be published than a similar study evincing negative findings. Research centres that have access to neuroimaging technologies are more likely to be tertiary centres to which atypical or 'difficult' cases are referred. Hence, their findings may have limited application in other settings. Also, this review itself has not addressed all the psychiatric syndromes that might involve bodily disturbance (e.g. anxiety) and the conclusions drawn here must be regarded as provisional.

In summary, many papers implicate association cortices and subcortical brain regions in the generation of abnormal bodily states, but offer limited insight into the specific pathophysiological mechanisms responsible. However, they shed enough light on the phenomenology of the embodied self for us to suggest that doctors should be especially cautious when interpreting physical phenomena that currently cannot be 'explained'. There are indeed perturbations of brain structure and function associated with many of these ill-understood states, so that our patients' words offer a first clue as to the systems that may be dysfunctional within their brains. These phenomenological accounts provide a potential signal of the current state of the embodied self.

'Our flesh is easily worn out; but in being so clearly subject to time and accident it reminds us of what we truly are. Our essence lies in what is most accidental about us - the time and place of our birth, our habits of speech and movement, the flaws and quirks of our bodies' (Gray, 2002: p. 144).

\section{Declaration of interest}

None

\section{References}

Alexander, G. E. \& Crutcher, M. D. (1990) Functional architecture of basal ganglia circuits: neural substrates of parallel processing. Trends in Neurosciences, 13, 266-271.

Aziz, Q., Schnitzler, A. \& Enck, P. (2000) Functional neuroimaging of visceral sensation. Journal of Clinical Neurophysiology, 17, 604-612.

Baldeweg, T., Spence, S., Hirsch, S. R., et al (1998) Gamma-band electroencephalographic oscillations in a patient with somatic hallucinations. Lancet, 352, 620-621.

Berrios, G. E. (1982) Tactile hallucinations: conceptual and historical aspects. Journal of Neurology, Neurosurgery and Psychiatry, 45, 285-293.

Blair, R. J. R. (2003) Neurobiological basis of psychopathy. British Journal of Psychiatry, 182, 5-7.

Butler, C. C., Evans, M., Greaves, D. et al (2004) Medically unexplained symptoms: the biopsychosocial model found wanting. Journal of the Royal Society of Medicine, 97, 219222.

Cahill, C. \& Frith, C. D. (1996) False perceptions or false beliefs? Hallucinations and delusions in schizophrenia. In Method in Madness: Case Studies in Cognitive Neuropsychology (eds P. W. Halligan \& J. C. Marshall), pp. 267-291. Hove: Psychology Press.

Calder, A. J., Lawrence, A. D. \& Young, A. W. (2001) Neuropsychology of fear and loathing. Nature Reviews Neuroscience, 2, 352-363.

Carey, P., Seedat, S., Warwick, J., et al (2004) SPECT imaging of body dysmorphic disorder. Journal of Neuropsychiatry and Clinical Neurosciences, 16, 357-359.

Chaudhuri, A. \& Behan, P. O. (2004) Fatigue in neurological disorders. Lancet, 363, 978-988.

Cleare, A. J., Messa, C., Rabiner, E. A., et al (2005) Brain 5-HT $1 \mathrm{~A}$ receptor binding in chronic fatigue syndrome measured 
using positron emission tomography and [11C] Way-100635. Biological Psychiatry, 57, 239-246.

Cook, D. B., Lange, G., Deluca, J., et al (2001) Relationship of brain MRI abnormalities and physical functional status in chronic fatigue syndrome. International Journal of Neuroscience, 107, $1-6$.

Cope, H., Pernet, A., Kendall, B., et al (1995) Cognitive functioning and magnetic resonance imaging in chronic fatigue. British Journal of Psychiatry, 167, 86-94.

Damasio, A. R. (2000) A neural basis of sociopathy. Archives of General Psychiatry, 57, 128-129.

De Lange, F. P., Kalkman, J. S., Bleijenberg, G., et al (2004) Neural correlates of the chronic fatigue syndrome - an fMRI study. Brain, 127, 1948-1957.

Derbyshire, S. W. G. (2003) Visceral afferent pathways and functional brain imaging. The Scientific World Journal, 3, 1065-1080.

Derbyshire, S. W. G., Whalley, M. G., Stenger, V. A., et al (2004) Cerebral activation during hypnotically induced and imagined pain. NeuroImage, 23, 392-401.

De Risio, S., De Rossi, G., Sarchiapone, M., et al (2004) A case of Cotard syndrome: 123 IBZM SPECT imaging of striatal D2 receptor binding. Psychiatry Research: Neuroimaging, 130, 109-112.

Dunckley, P., Wise, R. G., Aziz, Q., et al (2005) Cortical processing of visceral and somatic stimulation: differentiating pain intensity from unpleasantness. Neuroscience, 133, 533-542.

Franck, N., O'Leary, D. S., Flaum, M., et al (2002) Cerebral blood flow changes associated with Schneiderian first-rank symptoms in schizophrenia. Journal of Neuropsychiatry and Clinical Neuroscience, 14, 277-282.

Frank, G. K., Kaye, W. H., Meltzer, C. C., et al (2002) Reduced 5 HT2A receptor binding after recovery from anorexia nervosa. Biological Psychiatry, 52, 896-906.

Frank, G. K., Bailer, U. F., Henry, S. E., et al (2005) Increased dopamine D2/D3 receptor binding after recovery from anorexia nervosa measured by positron emission tomography and $\left[{ }^{11} \mathrm{C}\right]$ raclopride. Biological Psychiatry, 58, 908-912.

Frith, C. D. \& Done, D. J. (1989) Experiences of alien control in schizophrenia reflect a disorder in the central monitoring of action. Psychological Medicine, 19, 359-363.

Frith, C. D., Blakemore, S-J. \& Wolpert, D. M. (2000) Explaining the symptoms of schizophrenia: abnormalities in awareness of action. Brain Research Reviews, 31, 357-363.

Gabbay, V., Asnis, G. M., Bello, J. A., et al (2003) New onset of body dysmorphic disorder following frontotemporal lesion. Neurology, 61, 123-125.

Ganesan, V., Hunter, M. D. \& Spence, S. A. (2005) Schneiderian first-rank symptoms and right parietal hyperactivation: a replication using fMRI. American Journal of Psychiatry, 162, 1545.

Garcia-Campayo, J., Sanz-Carrillo, C., Baringo, T., et al (2001) SPECT scan in somatization disorder patients: an exploratory study of eleven cases. Australian and New Zealand Journal of Psychiatry, 35, 359-363.

Gardner-Thorpe, C. \& Pearn, J. (2004) The Cotard syndrome. Report of two patients: with a review of the extended spectrum of 'delire des negations'. European Journal of Neurology, 11 563-566.

Goldstein, J. A., Mena, I., Jouanne, E., et al (1995) The assessment of vascular abnormalities in late life chronic fatigue syndrome by brain SPECT: comparison with late life major depressive disorder. Journal of Chronic Fatigue Syndrome, 1, 55-79.

Gray, J. (2002) Straw Dogs. Thoughts on Humans and Other Animals. London: Granta Books.

Greco, A., Tannock, C., Brostoff, J., et al (1997) Brain MR in chronic fatigue syndrome. American Journal of Neuroradiology, 18, 1265-1269.

Hakala, M., Karlsson, H., Ruotsalainen, U., et al (2002) Severe somatization in women is associated with altered cerebral glucose metabolism. Psychological Medicine, 32, 1379-1385.

Hakala, M., Karlsson, H., Kurki, et al (2004) Volumes of the caudate nuclei in women with somatization disorder and healthy women. Psychiatry Research: Neuroimaging, 131, 71-78.

Hashioka, S., Monji, A., Sasaki, M., et al (2002) A patient with Cotard syndrome who showed an improvement in single photon emission computed tomography findings after successful treatment with antidepressants. Clinical Neuropharmacology, 25, 276-279.

Hayashi, H., Oshino, S., Ishikawa, J., et al (2004) Paroxetine treatment of delusional disorder, somatic type. Human Psychopharmacology: Clinical and Experimental, 19, 351-352.

Jones, A. K. P., Kulkarni, B. \& Derbyshire, S. W. G. (2003) Pain mechanisms and their disorders. British Medical Bulletin, 65, 83-93.

Ladabaum, U., Minoshima, S. \& Owyang, C. (2000) Pathobiology of visceral pain: molecular mechanisms and therapeutic implications: V. Central nervous system processing of somatic and visceral sensory signals. American Journal of Physiology - Gastrointestinal and Liver Physiology, 279, G1-G6.

Lange, G., Wang, S., Deluca, J., et al (1998) Neuroimaging in chronic fatigue syndrome. American Journal of Medicine, 105, 50S-53S.

Lange, G., Deluca, J., Maldjian, J. A., et al (1999) Brain MRI abnormalities exist in a subset of patients with chronic fatigue syndrome. Journal of the Neurological Sciences, 171, 3-7.

Lewis, D. H., Mayberg, H. S., Fischer, M. E., et al (2001) Monozygotic twins discordant for chronic fatigue syndrome: regional cerebral blood flow SPECT. Radiology, 219, 766-773.

Maeda, K., Yamamoto, Y., Yasuda, M., et al (1998) Delusions of oral parasitosis. Progress in Neuro-Psychopharmacology and Biological Psychiatry, 22, 243-248.

Mailis-Gagnon, A., Giannoylis, I., Downar, J., et al (2003) Altered central somatosensory processing in chronic pain patients with 'hysterical' anaesthesia. Neurology, 60, 1501-1507.

Marshall, J. C., Halligan, P. W., Fink, G. R., et al (1997) The functional anatomy of a hysterical paralysis. Cognition, 64, B1-B8.

Maruff, P., Wood, S. J., Velakoulis, D., et al (2005) Reduced volume of parietal and frontal association areas in patients with schizophrenia characterized by passivity delusions. Psychological Medicine, 35, 783-789.

Matsui, M., Yoneyama, E., Sumiyoshi, T., et al (2002) Lack of self-control as assessed by a personality inventory is related to reduced volume of supplementary motor area. Psychiatry Research: Neuroimaging, 116, 53-61.

Mullins, S. \& Spence, S. A. (2003) Re-examining thought insertion: semi-structured literature review and conceptual analysis. British Journal of Psychiatry, 182, 293-298.

Natelson, B. H., Cohen, J. M., Brassloff, I., et al (1993) A controlled study of brain magnetic resonance imaging in patients with the chronic fatigue syndrome. Journal of the Neurological Sciences, 120, 213-217

Okada, T., Tanaka, M., Kuratsune, H., et al (2004) Mechanisms underlying fatigue: a voxel-based morphometric study of chronic fatigue syndrome. BMC Neurology, 4, 14.

Ota, M., Mizukami, K., Katano, T., et al (2003) A case of delusional disorder, somatic type with remarkable improvement of clinical symptoms and single photon emission tomography findings following modified electroconvulsive therapy. Progress in Neuro-Psychopharmacology and Biological Psychiatry, 27, 881-884.

Peyron, R., Laurent, B. \& Garcia-Larrea, L. (2000) Functional imaging of brain responses to pain. A review and metaanalysis. Neurophysiologie Clinique, 30, 263-288.

Phan, K. L., Wager, T., Taylor, S. F., et al (2002) Functional neuroanatomy of emotion: a meta-analysis of emotion activation studies in PET and fMRI. NeuroImage, 16, 331348

Rauch, S. L., Phillips, K. A., Segal, E., et al (2003) A preliminary morphometric magnetic resonance imaging study of regional brain volumes in body dysmorphic disorder. Psychiatry Research: Neuroimaging, 122, 13-19.

Rosendal, M., Olesen, F. \& Fink, P. (2005) Management of medical unexplained symptoms. BMJ, 330, 4-5.

Schneider, K. (1974) Primary and secondary symptoms in schizophrenia. In Themes and Variations in European Psychiatry (eds S. R. Hirsch \& M. Shepherd), pp. 40-46. Bristol: John Wright.

Schnitzler, A., Volkmann, J., Enck, P., et al (1999) Different cortical organization of visceral and somatic sensation in humans. European Journal of Neuroscience, 11, 305-315. 
Seeger, G., Braus, D. F., Ruf, M., et al (2002) Body image distortion reveals amygdala activation in patients with anorexia nervosa - a functional magnetic resonance imaging study. Neuroscience Letters, 326, 25-28.

Shergill, S. S., Cameron, L. A., Brammer, M. J., et al (2001) Modality specific neural correlates of auditory and somatic hallucinations. Journal of Neurology, Neurosurgery and Psychiatry, 71, 688-690.

Siessmeier, T., Nix, W. A., Hardt, J., et al (2003) Observer independent analysis of cerebral glucose metabolism in patients with chronic fatigue syndrome. Journal of Neurology, Neurosurgery and Psychiatry, 74, 922-928.

Spence, S. A. (1999) Hysterical paralyses as disorders of action. Cognitive Neuropsychiatry, 4, 203-226.

Spence, S. A. (2001) Alien control: from phenomenology to cognitive neurobiology. Philosophy, Psychiatry and Psychology, 8, 163-172.

Spence, S. A. (2006) The cognitive executive is implicated in the maintenance of psychogenic movement disorders. In Psychogenic Movement Disorders: Neurology and Neuropsychiatry (eds M. Hallett, S. Fahn, J. Jankovic, et al), pp. 222-229. Philadelphia, PA: American Academy of Neurology Press.

Spence, S. A. \& Frith, C. D. (1999) Towards a functional anatomy of volition. Journal of Consciousness Studies, 6, 11-29.

Spence, S. A. \& Halligan, P. W. (2002) Pathologies of Body, Self and Space. Hove: Psychology Press.

Spence, S. A., Brooks, D. J., Hirsch, S. R., et al (1997) A PET study of voluntary movement in schizophrenic patients experiencing passivity phenomena (delusions of alien control). Brain, 120, 1997-2011.

Spence, S. A., Crimlisk, H. L., Cope, H., et al (2000) Discrete neurophysiological correlates in prefrontal cortex during hysterical and feigned disorder of movement. Lancet, 355, 1243-1244.

Stone, J. \& Zeman, A. (2001) Hysterical conversion - a view from clinical neurology. In Contemporary Approaches to the Study of Hysteria: Clinical and Theoretical Perspectives (eds P. W. Halligan, C. Bass \& J. C. Marshall), pp. 102-125. Oxford: Oxford University Press.

Talley, N. (1998) Scope of the problem of functional digestive disorders. European Journal of Surgery, 582 (suppl.), 35-41.

Tiihonen, J., Kuikka, J., Viinamaki, H., et al (1995) Altered cerebral blood flow during hysterical paraesthesia. Biological Psychiatry, 37, 134-135.

Uher, R. \& Treasure, J. (2005) Brain lesions and eating disorders. Journal of Neurology, Neurosurgery and Psychiatry, 76, 852857.

Uher, R., Murphy, T., Friederich, H-C., et al (2005) Functional neuroanatomy of body shape perception in healthy and eatingdisordered women. Biological Psychiatry, 58, 990-997.

Vogt, B. A. (2005) Pain and emotion interactions in subregions of the cingulate gyrus. Nature Reviews Neuroscience, 6, 533-544.

Vuilleumier, P., Chicherio, C., Assal, F., et al (2001) Functional neuroanatomical correlates of hysterical sensorimotor loss. Brain, 124, 1077-1090.

Wada, T., Kawakatsu, S., Komatani, A., et al (1999) Possible association between delusional disorder, somatic type and reduced regional cerebral blood flow. Progress in NeuroPsychopharmacology and Biological Psychiatry, 23, 353-357.

Werring, D. J., Weston, L., Bullmore, E. T., et al (2004) Functional magnetic resonance imaging of the cerebral response to visual stimulation in medically unexplained visual loss. Psychological Medicine, 34, 583-589.

Yazici, K. M. \& Kostakoglu, L. (1998) Cerebral blood flow changes in patients with conversion disorder. Psychiatry Research: Neuroimaging, 83, 163-168.

\section{MCQs}

1 The following are methods used to image brain function:

a structural MRI

b computed tomography

c functional MRI

d SPECT

e air encephalography.

2 The following disorders have been associated with abnormalities of temporo-parietal function:

a delusional parasitosis

b passivity phenomena

c conversion disorder

d dysmorphophobia

e Cotard's delusion.

3 The caudate nucleus is:

a part of the striatum

b a component of the 'executive' circuit

c implicated in some studies of somatisation disorder

$\mathrm{d}$ involved in the processing of disgust

e implicated in some studies of chronic fatigue syndrome.

4 The frontal lobes:

a lie posterior to the central sulcus

$\mathrm{b}$ are engaged in planning and programming actions

c contain loops projecting to and from the thalamus

$\mathrm{d}$ are perfectly symmetrical

e have centres involved in working memory.

5 Clinically useful structural data may be obtained from the following:

a brain computed tomography

b brain MRI

c EEG

d fMRI

e Wada testing. 